\title{
Creep Behaviour of Woven Roving GRP Beam in Three- Point Bending
}

\author{
Shuang-yin Zhang \\ Institute of Mechanics, Chinese Academy of Sciences, Beijing 100080, People's Republic \\ of China \\ $\&$ \\ Bing Zhao \\ Institute of Geophysics, Chinese Academy of Sciences, Beijing 100080, People's Republic \\ of China
}

\begin{abstract}
The creep and relaxation behaviour of laminated glass fibre reinforced plastics (GRP) in three-point bending were studied both experimentally and analytically. Creep and relaxation experiments were carried out on eight types of specimens, consisting of glass fibre fabric reinforced epoxy beams. While the bending deflexion and creep strains were measured in the creep tests, the load and relaxation strain were recorded in the relaxation tests. Marked creep effects were seen in the tests, where the environment temperature was $50^{\circ} \mathrm{C}$ and the period of the measurement was $60 \mathrm{~min}$. An attempt to predict the creep deflexion and relaxation behaviour was made. The transverse shear effect on creep deflexion was taken into account. The predicted results were compared with experimental ones. They were found to be in reasonable agreement, but the linearization assumption, upon which the relaxation behaviour analysis was based, appears to lead to larger inaccuracies in the results.
\end{abstract}

\section{NOTATION}

$a$

$A$

$A_{1}$

$b$
Half span of the beam specimen (Fig. 1)

Material constant (eqn (1))

Constant (eqn (26))

Width of the beam (Fig. 1)

Composite Structures 0263-8223/89/\$03.50 (C) 1989 Elsevier Science Publishers Ltd, England. Printed in Great Britain 


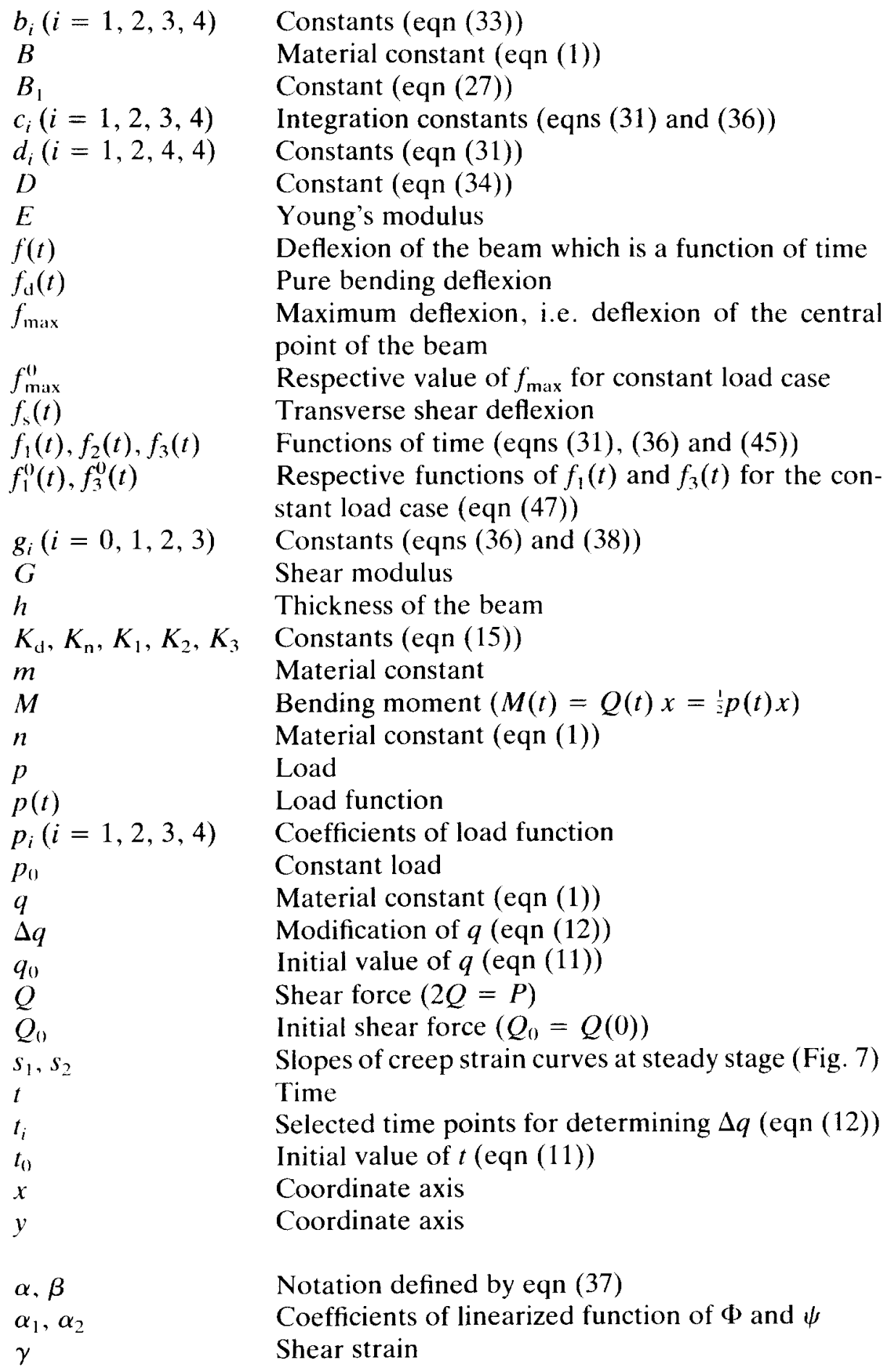




$\begin{array}{ll}\varepsilon & \begin{array}{l}\text { Direct strain } \\ \text { Direct strain at the top surface of the beam, } \\ \varepsilon_{\mathrm{h}}\end{array} \\ \varepsilon_{\mathrm{h}}=\left.\varepsilon\right|_{y=\mathrm{h}} \\ \varepsilon_{i} & \begin{array}{l}\text { Strains at the selected times, } t_{i}, \text { for determining } \Delta q \\ \text { (eqn (12)) }\end{array} \\ \varepsilon_{\mathrm{r}_{1}}, \varepsilon_{\mathrm{r}_{2}} & \text { As shown in Fig. } 7 \\ \varepsilon_{\mathrm{T}} & \text { Direct strain at steady creep stage } \\ \varepsilon_{0} & \text { Initial value of } \varepsilon \text { (eqn (11)) } \\ \varepsilon^{*} & \text { Equivalent strain } \\ \sigma & \text { Direct stress } \\ \sigma_{\mathrm{h}} & \text { Direct stress at the top surface of the beam } \\ \sigma_{\mathrm{hC}} & \text { Direct stress of the top surface at time C (see Fig. 7) } \\ \sigma_{\mathrm{T}} & \text { Direct stress at steady creep stage } \\ \sigma_{0} & \text { Initial value of } \sigma \text { (eqn (11)) } \\ \sigma^{*} & \text { Equivalent stress } \\ \tau & \text { Shear stress } \\ \Phi(t), \psi(t) & \text { Functions of time (eqn (2)) }\end{array}$

\section{INTRODUCTION}

Glass fibre reinforced plastics (GRP) have long been used and are being increasingly applied to engineering structures and constructions. They have the advantageous properties of high specific strength and modulus. But they show remarkable viscoelastic effects when loaded by bending, shearing and torsional forces and under off-axial tension in respect to the fibre direction. In order to ensure that the structural components of GRP are reliable in service, the designer should know their creep behaviour well and take it into account in design. The relaxation behaviour of chopped strand mat (CSM)-GRP was experimentally studied in the previous paper. ${ }^{1}$ The research described in the present paper is concerned with creep and relaxation behaviour of glass fibre reinforced plastics in threepoint bending. Eight types of GRP beam specimen were tested.

The creep of composite materials is nonlinear in most cases, even when the deformation is very small, unless the duration of measurement is very short. The creep problem of a three-point bending beam is of particular complication, since both stress and strain are functions of coordinate as well as of time. So, few papers are seen in literature dealing with analysis of this problem. In the present paper, an attempt is made to predict creep deflexion and relaxation strain, and the transverse shear effect of the composite beam is taken into consideration. The typical creep and 
relaxation behaviour of one kind of specimen were analysed. The predicted results were compared with experimental ones. The agreement between them is reasonable.

\section{EXPERIMENTAL INVESTIGATION}

The three-point bending test represents a typical loading case of many structures. The transverse shear creep behaviour which characterizes the laminated composites can be examined through this test scheme.

\subsection{Materials and specimens}

The tested materials are eight types of laminated sheet made of glass fibre fabric reinforced epoxy resin. The specimens are categorized into eight groups according to their warp to weft ratio and lay-up angle. The configuration and glass content of the specimens are shown in Table 1 . The nominal dimensions of the specimens are $80 \mathrm{~mm} \times 15 \mathrm{~mm} \times(3-5) \mathrm{mm}$.

TABLE 1

Categories of the Specimens

\begin{tabular}{|c|c|c|c|c|c|c|c|c|}
\hline Group number & I & II & III & IV & V & VI & VII & VIII \\
\hline Warp/weft ratio & $1: 1$ & $1: 1$ & $4: 1$ & $4: 1$ & $4: 1$ & $7: 1$ & $7: 1$ & $7: 1$ \\
\hline Lay-up angle & $0^{\circ}$ & $\pm 45^{\circ}$ & $0^{\circ}$ & $90^{\circ}$ & $\pm 45^{\circ}$ & $0^{\circ}$ & $90^{\circ}$ & \pm 45 \\
\hline Number of layers & 11 & 11 & 11 & 11 & 11 & 7 & 7 & 7 \\
\hline $\begin{array}{l}\text { Volume fraction of fibre } \\
(\%)\end{array}$ & $29 \cdot 3$ & $29 \cdot 4$ & $33 \cdot 1$ & $31 \cdot 1$ & $26 \cdot 9$ & 29.9 & $31 \cdot 0$ & $26 \cdot 0$ \\
\hline
\end{tabular}

\subsection{Experiments}

The experimental set-up and the dimensions of the specimen are schematically shown in Fig. 1. The tests were conducted by using the INSTRON 1195 testing machine. The temperature in the testing chamber is $50^{\circ} \mathrm{C}$. When the load, $p$, is kept constant the variations of deflexion and strains with time are measured and the test is then called the creep test. If the deflexion of the beam specimen is held constant, the load and strain are measured as functions of time, the test is called the relaxation test. Before the tests, some preliminary tests were conducted to measure Young's modulus $E$ and the shear modulus $G$ under the temperature of $50^{\circ} \mathrm{C}$. Two strain gauges were used to measure creep and relaxation strains. They were stuck on both sides of the beam and positioned at the centre and 1/4 span of the beam respectively. 


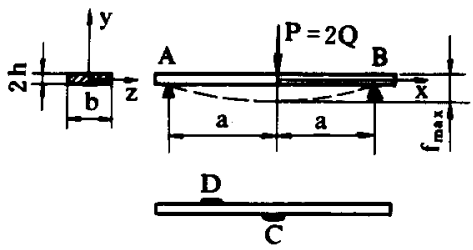

Fig. 1. Experimental scheme and specimen configuration.

The test procedure is as follows. At first, the specimen is warmed to $50^{\circ} \mathrm{C}$ in the testing chamber and kept at $50^{\circ} \mathrm{C}$ for 20 min until the bending load was applied. The bending load should be applied as fast as possible to reduce the creep effect in the loading process, but impact must be avoided. When the strain reaches a predetermined value, which is below $6000 \times 10^{-6} \mathrm{~mm} / \mathrm{mm}$, and the load $p$ (or the deflexion of the central point of the beam) is kept constant, the strains and the deflexion (or load for the relaxation test) are measured. The creep duration was $60 \mathrm{~min}$ or longer.

\subsection{Experimental results and discussion}

The creep strain and deflexion curves of the eight specimens are illustrated in Figs 2 and 3, respectively. It is obvious that the specimens with a $45^{\circ}$ lay-up angle exhibit the most remarkable creep effect, because in addition

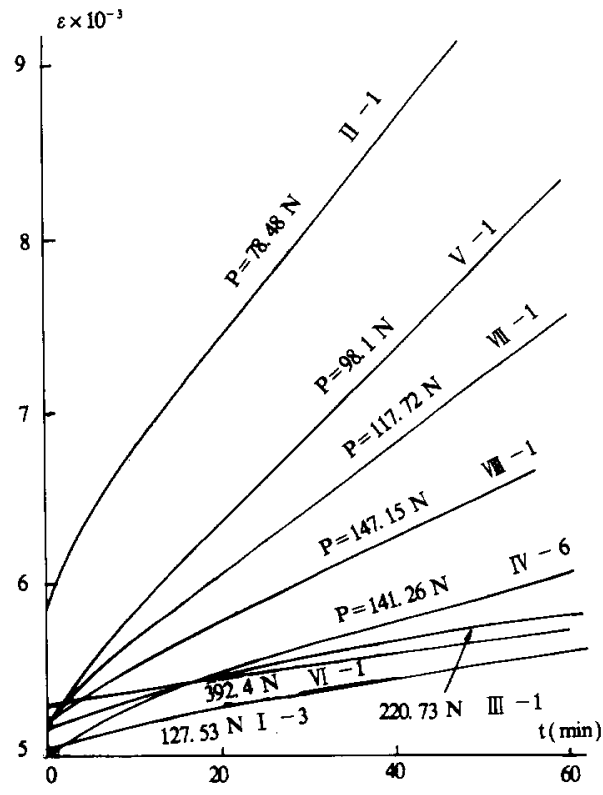

Fig. 2. Creep strain curves.

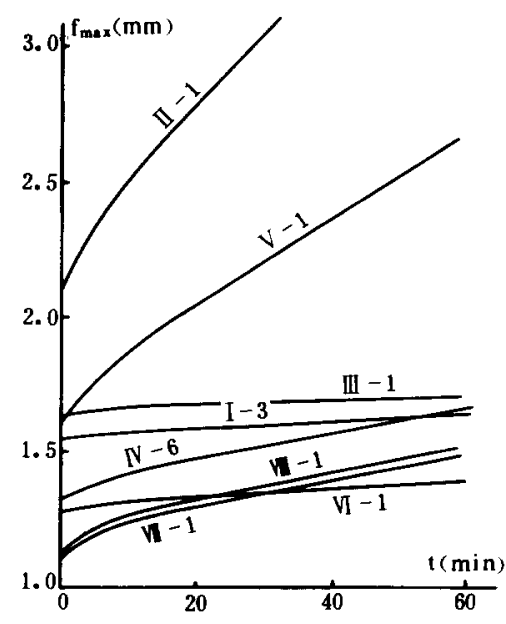

Fig. 3. Creep deflexion curves. 


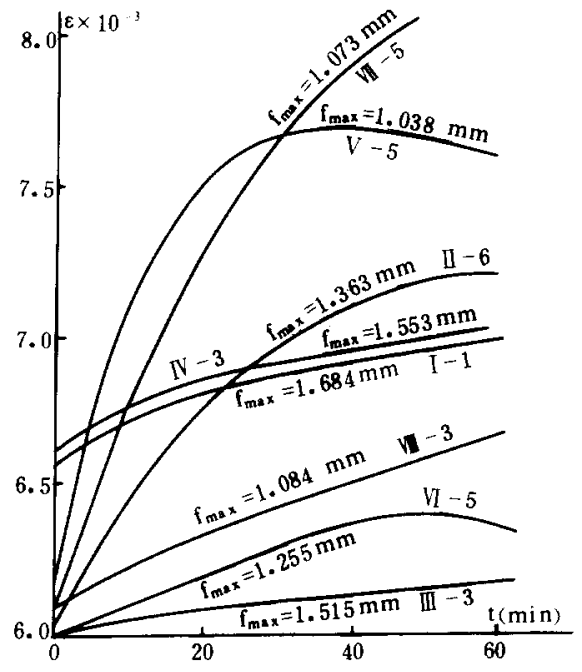

Fig. 4. Relaxation strain curves.

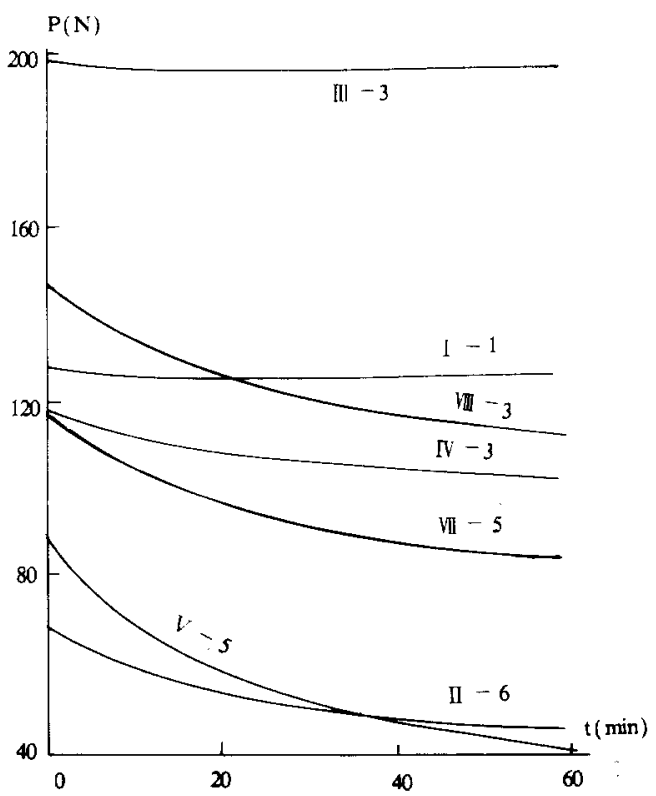

Fig. 5. Relaxation load curves of seven specimens. 


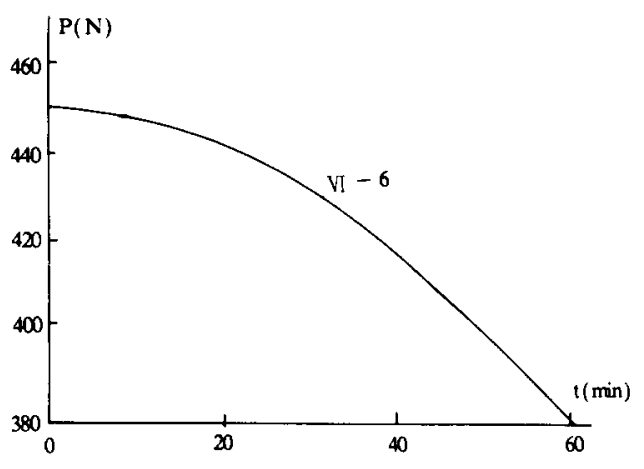

Fig. 6. Relaxation load curve of specimen VI-5.

to transverse shear deformation, the in-plane shear deformation gives large contributions to the creep strain and displacement. Comparing deflexion $f(t)$ and strain $\varepsilon(t)$ curves of $0^{\circ}$ specimens with those of $90^{\circ}$ specimens reveals that the former have less creep effect than the latter. Among the $0^{\circ}$ specimens of the three different warp/weft ratios, the $7: 1$ specimens have the least creep effect. Figures 4, 5 and 6 are the relaxation strain and load curves. It can be seen that all the load curves decrease monotonically with time. But as for the strain curve, while most curves increase monotonically with time in the duration of measurement, the curves of specimens of V-5 and VI-5 increase with time at the early stage and after reaching their maximum values tend to decrease. This reveals the complexity of the problem.

\section{THEORETICAL PREDICTIONS}

\subsection{Basic assumptions}

In the three-point bending beam undergoing creep and relaxation tests, the strains and stresses are all functions of time and coordinates, so the analyses are very complicated. To simplify the calculations the following assumptions were adopted:

(1) The small strain and small deflexion assumption: For all tests, the largest strain is smaller than $2 \%$ and the largest ratio of deflexion to span is smaller than $4 \%$.

(2) The plane cross-section assumption: That is, the cross-section of the beam is assumed to remain flat after deformation, but no longer perpendicular to the central surface of the beam. 
(3) The material behaviour in tension is the same as that in compression, so both cases can be analysed by using the same constitutive equation.

\subsection{Creep equations}

The deflexion in both creep and relaxation tests can be divided into two parts: pure bending deflexion and transverse shear deflexion. The following equation was used to calculate the pure bending deflexion in the creep test:

$$
\varepsilon=\frac{\sigma}{E}+A \sigma^{m}\left(1-\mathrm{e}^{-q t}\right)+B \sigma^{n} t
$$

The first term on the right side of eqn (1) refers to the elastic strain. The second and third terms are for the transient and steady stages respectively. For relaxation, the pure bending deflexion was analysed by using the following generalized creep equation: ${ }^{2}$

$$
\ddot{\varepsilon}+q \dot{\varepsilon}=\frac{\ddot{\sigma}}{E}+\left(\dot{\Phi}+\psi+\frac{q \dot{\sigma}}{E}\right)+q \psi
$$

where $\Phi=A \sigma^{m}$ and $\psi=B \sigma^{n}$.

As for the creep equation of transverse shear creep effect, it was assumed that the transverse shear deflexion could be approximately estimated by a one-term equation which corresponds to the steady stage of creep. The equivalent stress and equivalent strain for a pure shear stress state are given as

$$
\begin{aligned}
& \sigma^{*}=\sqrt{3} \tau \\
& \varepsilon^{*}=\frac{1}{\sqrt{3}} \gamma
\end{aligned}
$$

The relationship between $\sigma^{*}$ and $\varepsilon^{*}$ is

$$
\varepsilon^{*}=B \sigma^{* n}
$$

Then

$$
\dot{\gamma}=3^{(n+1) / 2} B \tau^{n}
$$




\subsection{Experimental determination of the constants in equation (1)}

The analysis proceeds as follows. The five constants of eqn (1), $A, B, m, n$ and $q$, are determined experimentally first, then, by using eqns (1) and (6) the creep deflexion of the beam is calculated. Finally, the relaxation strain and deflexion with eqns (2) and (6) are analysed.

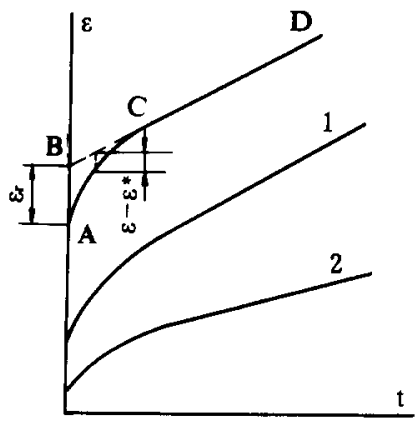

Fig. 7. Creep strain curves for determining the constants in eqn (1).

The two constants $B$ and $n$ in eqn (1) were determined by referring to Fig. 7 and using the following formulae:

$$
\begin{aligned}
& n=\ln \left(s_{1} / s_{2}\right) / \ln \left(x_{1} / x_{2}\right) \\
& B=s_{1} \sigma_{\mathrm{hT}}^{-n}
\end{aligned}
$$

where $s_{1}$ and $s_{2}$ are the slopes of the creep curves at the steady stage and $x_{1}$ and $x_{2}$ are the coordinates of the two strain gauges on the bending specimen. Subscript $T$ refers to the steady creep stage.

From the measurements of the transient creep stage, the constants $A$ and $m$ were calculated by using the following formulae:

$$
m=\frac{\ln \left(\varepsilon_{\mathrm{r}_{1}} / \varepsilon_{\mathrm{r}_{2}}\right)}{\ln \left(x_{1} / x_{2}\right)}
$$

where $\varepsilon_{\mathrm{r}_{i}}$ are as shown in Fig. 7.

$$
A=\varepsilon_{\mathrm{r}_{1}} \sigma_{\mathrm{hT}}^{-m}
$$

The constant $q$ was determined through a quasi-least-square method. An initial value $q_{0}$ was approximated by substituting an estimated value of stress $\sigma_{0}$ into eqn (1). $\sigma_{0}$ was selected as the mean value of the elastic stress 
and $\sigma_{\mathrm{hC}}$, and the corresponding time $t_{0}$ was assumed to be half the time duration of the transient stage.

$$
\varepsilon_{0}=\frac{\sigma_{0}}{E}+A \sigma_{0}^{m}\left(1-\mathrm{e}^{-q_{0} t_{0}}\right)+B \sigma_{0}^{n} t_{0}
$$

where $\varepsilon_{0}$ is the measured strain at $t_{0}$.

The modification of $q$ can be calculated through the formula

$$
\Delta q=\frac{\sum_{1}\left(\varepsilon_{0}-\varepsilon_{i}\right) \mathrm{e}^{-q_{\| 1} t_{i}} t_{i}}{\left(\sum \mathrm{e}^{-2 q_{0} t_{i}} t_{i}^{2}\right) A \sigma_{0}^{n}}
$$

Thus, the approximate value of $q$ can be obtained as

$$
q=q_{0}+\Delta q
$$

\subsection{Solution for the creep deflexion}

\subsubsection{The deflexion for pure bending}

Based on the solution from Ref. 3 , the pure bending creep deflexion $f_{\mathrm{d}}$ can be obtained by integration of the following equation:

$$
h \frac{\mathrm{d}^{2} f_{\mathrm{d}}}{\mathrm{d} x^{2}}=K_{1} M+K_{2} M^{m}+K_{3} M^{n}
$$

where $M$ is the bending moment and is equal to $Q x$,

$$
\begin{aligned}
& K_{1}=3 /\left(2 E b h^{2}\right) \\
& K_{2}=A\left(\frac{K_{\mathrm{n}}}{2 b h^{2}}\right)^{m}\left\{\frac{\left(1+K_{\mathrm{d}}\right)^{m+1}}{K_{\mathrm{d}}(1+m)}-\frac{\left(1+K_{\mathrm{d}} \mathrm{e}^{-q t}\right)^{m+1}}{K_{\mathrm{d}}(m+1)}\right\} \\
& K_{3}=B\left(\frac{K_{\mathrm{n}}}{2 b h^{2}}\right)^{n}\left\{t+\frac{n K_{\mathrm{d}}}{q}\left(1-\mathrm{e}^{-q t}\right)\right\} \\
& K_{\mathrm{n}}=(2 n+1) / n \\
& K_{\mathrm{d}}=\left(3-K_{\mathrm{n}}\right) / K_{\mathrm{n}}
\end{aligned}
$$


Upon integration and using boundary conditions, the following are obtained;

$$
\begin{aligned}
f_{\mathrm{d}}= & \frac{K_{1} Q}{6 h} x^{3}+\frac{K_{2} Q^{m}}{(m+1)(m+2) h} x^{m+2}+\frac{K_{3} Q^{n}}{h(n+1)(n+2)} x^{n+2} \\
& -\frac{x}{h}\left[K_{1} Q \frac{a^{2}}{2}+K_{2} Q^{m} \frac{a^{m+1}}{m+1}+\frac{K_{3} Q^{n}}{n+1} a^{n+1}\right]
\end{aligned}
$$

\subsubsection{The transverse shear deflexion}

The creep stress at the steady stage can be expressed $a^{3}$

$$
\sigma_{\mathrm{T}}=\frac{2 n+1}{n}\left(\frac{y}{h}\right)^{1 / n} \frac{M}{2 b h^{2}}
$$

Making use of the equilibrium equation and the boundary conditions, the following expression of shear stress is obtained:

$$
\tau_{\mathrm{T}}=\frac{1+2 n}{1+n} \frac{Q}{2 b h}\left[1-\left(\frac{y}{h}\right)\right]^{1+1 / n}
$$

Substituting the above equation into eqn (6) and letting $y=0$, the shear strain at the middle surface of the beam is derived as

$$
\gamma_{y=0}=\frac{3}{4} \frac{Q}{b h G}+3^{(n+1) / 2} B\left(\frac{1+2 n}{1+n} \frac{Q}{2 b h}\right)^{n} t
$$

According to the Timoshenko beam theory, the slope of the deflexion curve caused by the transverse shear force is equal to the shear strain of the beam at the central surface, i.e.

$$
\frac{\mathrm{d} f_{\mathrm{s}}}{\mathrm{d} x}=\left.\gamma\right|_{y=0}
$$

Thus, the transverse shear deflexion was obtained through a simple integration

$$
f_{\mathrm{s}}=\frac{3}{2} \frac{Q x}{2 b h G}+3^{(n+1) / 2} B\left(\frac{1+2 n}{1+n} \frac{Q}{2 b h}\right)^{n} x t
$$


The total deflexion of the central point of the beam is

$$
f_{\max }=\left\{f_{\mathrm{s}}+f_{\mathrm{d}}\right\}_{x=\mathrm{r}}
$$

Thus

$$
\begin{aligned}
f_{\max }= & \frac{K_{1} Q}{3 h} a^{3}+\frac{K_{2} Q^{m}}{h(m+2)} a^{m+2}+\frac{K_{3} Q^{n}}{h(n+2)} a^{n+2} \\
& +\frac{3}{2} \frac{Q a}{2 b h G}+3^{(n+1) / 2} B\left(\frac{1+2 n}{1+n} \frac{Q}{2 b h}\right)^{n} a t
\end{aligned}
$$

\subsection{Solution for the relaxation}

\subsubsection{Calculation procedure}

Since the solution of the relaxation of the three-point bending beam is more complicated, it had to be dealt with through a further approximation. The method was not to take $f_{\max }=$ const. as the initial condition and to solve the creep equation to obtain $Q(t)$ and $\varepsilon(t)$, but to take the measured load curve of $Q(t)$ as the known condition and calculate $\varepsilon(t)$ and $f_{\max }(t)$.

\subsubsection{Bending load function}

The measured load curve $P(t)$ was fitted with the third-order polynomial:

$$
P(t)=P_{1}+P_{2} t+P_{3} t^{2}+P_{4} t^{3}
$$

Using the least-square method, the coefficients of this function were determined.

\subsubsection{Solution of strain function}

The non-linear eqn (2) is extraordinarily difficult to handle. It was assumed that when $m$ and $n$ were not far from unity the two functions $\Phi=A \sigma^{m}$ and $\psi=B \sigma^{n}$ could be replaced by $\Phi=\alpha_{1} \sigma$ and $\psi=\alpha_{2} \sigma$, respectively. Thus, eqn (2) becomes

$$
\ddot{\sigma}+A_{1} \dot{\sigma}+B_{1} \sigma=E(\ddot{\varepsilon}+q \dot{\varepsilon})
$$

where

$$
A_{1}=\left(\alpha_{1}+\alpha_{2}\right) E+q
$$

and

$$
B_{1}=\alpha_{2} q E
$$


Multiplying both sides of eqn (25) by by and integrating it through the thickness of the beam, the following is obtained:

$$
\ddot{\varepsilon}_{\mathrm{h}}+q \dot{\varepsilon}_{\mathrm{h}}=\frac{3}{2 E b h^{2}}\left(\ddot{M}+A_{1} \dot{M}+B_{1} M\right)
$$

where

$$
M=\int_{-h}^{h} b \sigma y \mathrm{~d} y
$$

and

$$
\varepsilon=\sigma_{\mathrm{h}} \frac{y}{h}
$$

$\varepsilon_{\mathrm{h}}$ denotes the strain of the top surface of the beam and eqn (30) is based upon the flat cross-section assumption.

Substituting the loading function (24) into eqn (28) and remembering $M=Q(t) x=\frac{1}{2} P(t) x$, the strain function $\varepsilon_{\mathrm{h}}(t)$ is obtained:

$$
\varepsilon_{\mathrm{h}}(t)=\left(c_{1}+c_{2} \mathrm{e}^{-q t}+d_{1} t+d_{2} t^{2}+d_{3} t^{3}+d_{4} t^{4}\right) x=f_{1}(t) x
$$

where

$$
\begin{aligned}
& d_{1}=\frac{b_{1}}{q}-\frac{b_{2}}{q^{2}}+\frac{2 b_{3}}{q^{3}}-\frac{6 b_{4}}{q^{4}} \\
& d_{2}=\frac{b_{2}}{2 q}-\frac{b_{3}}{q^{2}}+\frac{3 b_{4}}{q^{3}} \\
& d_{3}=\frac{b_{3}}{3 q}-\frac{b_{4}}{q^{2}} \\
& d_{4}=\frac{b_{4}}{4 q}
\end{aligned}
$$

and

$$
\begin{aligned}
& b_{1}=D\left(2 P_{3}+A_{1} P_{2}+B_{1} P_{1}\right) \\
& b_{2}=D\left(6 P_{4}+4 A_{1} P_{3}+B_{1} P_{2}\right) \\
& b_{3}=D\left(3 A_{1} P_{4}+B_{1} P_{3}\right) \\
& b_{4}=D B_{1} P_{4}
\end{aligned}
$$


and

$$
D=3 /\left(4 E b h^{3}\right)
$$

The two integration constants $c_{1}$ and $c_{2}$ in eqn (31) can be determined by using the measured curve of relaxation, $\varepsilon_{\mathrm{h}}(t)$. From eqn (31):

$$
\varepsilon(t)=\frac{y}{h} \varepsilon_{\mathbf{h}}(t)=\frac{1}{h} f_{1}(t) x y
$$

\subsubsection{Solution of stress}

Substituting $\varepsilon(t)$ of eqn (35) into eqn (25),

$$
\sigma(t)=\left(c_{3} \mathrm{e}^{\alpha t}+c_{4} \mathrm{e}^{\beta t}+g_{0}+g_{1} t+g_{2} t^{2}+g_{3} t^{3}\right) x y=f_{2}(t) x y
$$

where

$$
\begin{aligned}
& \alpha=-\frac{A_{1}}{2}+\frac{1}{2} \sqrt{A_{1}^{2}-4 B_{1}} \\
& \beta=-\frac{A_{1}}{2}-\frac{1}{2} \sqrt{A_{1}^{2}-4 B_{1}}
\end{aligned}
$$

and

$$
\begin{aligned}
& g_{0}=\frac{E}{h B_{1}}\left\{b_{1}-b_{2} \frac{A_{1}}{B_{1}}-2 b_{3}\left[\frac{1}{B_{1}}-\left(\frac{A_{1}}{B_{1}}\right)^{2}\right]-6 b_{4}\left[\frac{A_{1}}{B_{1}}-\left(\frac{A_{1}}{B_{1}}\right)^{3}\right]\right\} \\
& g_{1}=\frac{E}{h B_{1}}\left\{b_{2}-\frac{2 b_{3} A_{1}}{B_{1}}-6 b_{4}\left[\frac{1}{B_{1}}-\left(\frac{A_{1}}{B_{1}}\right)^{2}\right]\right\} \\
& g_{2}=\frac{E}{h B_{1}}\left(b_{3}-\frac{3 b_{4} A_{1}}{B_{1}}\right) \\
& g_{3}=E b_{4} /\left(h B_{1}\right)
\end{aligned}
$$

The two integration constants $c_{3}$ and $c_{4}$ in eqn (36) are determined by using the following two initial conditions:

(1) When $t=0$, the stress $\sigma(t)$ equals the initial elastic stress value.

(2) Substituting $\dot{\sigma}$ and $\ddot{\sigma}$ from eqn (36) and $\dot{\varepsilon}$ and $\ddot{\varepsilon}$ from eqn (35) into eqn (25) and setting $t=0$, the second condition for determining the two constants $c_{3}$ and $c_{4}$ is obtained. 
When the stress solution of eqn (36) is known, the shear stress $\tau(t)$ is derived as follows:

$$
\tau(t)=f_{2}(t) \frac{h^{2}-y^{2}}{2}
$$

\subsubsection{Solution for bending deflexion}

The formula for calculating the deflexion of the beam caused by pure bending moment can be derived from the following equation:

$$
\frac{\varepsilon_{\mathrm{h}}}{h}=\frac{\mathrm{d}^{2} f_{\mathrm{d}}}{\mathrm{d}^{2} x}
$$

Upon integration and using the boundary conditions, the deflexion of the central point was obtained as:

$$
f_{\mathrm{d} \max }=\frac{a^{3}}{3 h} f_{1}(t)
$$

The transverse shear deflexion was derived through the following procedure.

Substituting eqn (39) into eqn (6) yields

$$
\dot{\gamma}=3^{(n+1) / 2} B f_{2}^{n}(t)\left(\frac{h^{2}}{2}-\frac{y^{2}}{2}\right)^{n}
$$

Integrating eqn (42) and setting initial conditions gives

$$
\begin{aligned}
\gamma= & 3^{(n+1) / 2} B\left(\frac{h^{2}-y^{2}}{2}\right)^{n} \frac{\left\{f_{2}(t)\right\}^{n+1}}{(n+1)\left(c_{3} \alpha \mathrm{e}^{o f}+c_{4} \beta \mathrm{e}^{\beta t}+g_{1}+2 g_{2} t+3 g_{3} t^{2}\right)} \\
& -3^{(n+1) / 2} B\left(\frac{h^{2}-y^{2}}{2}\right)^{n} \frac{\left(c_{3}+c_{4}+g_{0}\right)^{n+1}}{(n+1)\left(c_{3} \alpha+c_{4} \beta+g_{1}\right)}+\frac{3 Q_{0}}{4 h b G}\left(1-\frac{y^{2}}{h^{2}}\right)
\end{aligned}
$$

By virtue of eqn (20), the transverse shear deflexion of the central point of the beam was obtained as

$$
f_{\text {smax }}=\frac{3 Q_{0} a}{4 h b G}+3^{(n+1) / 2} \frac{B a}{(n+1)} f_{3}(t)\left(\frac{h^{2}}{2}\right)^{n}
$$


where

$$
f_{3}(t)=\frac{f_{2}^{n+1}(t)}{\left(c_{3} \alpha \mathrm{e}^{\alpha t}+c_{4} \beta \mathrm{e}^{\beta t}+g_{1}+g_{2} t+3 g_{3} t^{2}\right)}-\frac{\left(c_{3}+c_{4}+g_{0}\right)^{n+1}}{\left(c_{3} \alpha+c_{4} \beta+g_{1}\right)}
$$

Thus, the total deflexion is

$$
f_{\max }=\frac{a^{3}}{3 h} f_{1}(t)+\frac{3 Q_{0} a}{4 b h G}+3^{(n+1) / 2} \frac{B a}{n+1} f_{3}(t)\left(\frac{h^{2}}{2}\right)^{n}
$$

\subsubsection{Creep deflexion under constant load}

If $P(t)$ is set constant and equal to $P_{0}$, and, following the procedure described above, the formula of creep deflexion is obtained as follows:

$$
f_{\max }^{\prime \prime}=\frac{a^{3}}{3 h} f_{1}^{0}(t)+\frac{3 Q_{0}}{4 b h G}+3^{(n+1) / 2} \frac{B a}{n+1} f_{3}^{0}(t)\left(\frac{h^{2}}{2}\right)^{n}
$$

where $f_{\max }^{0}, f_{1}^{0}(t)$ and $f_{3}^{0}(t)$ are the formulae corresponding to eqns (47), (31) and (45) for the case of constant load. Equation (47) is the creep deflexion of the solution of eqn (2). The predicted results of eqn (23) and eqn (47) are compared in the following section.

\subsection{Numerical results}

The creep deflexion of specimen II-1 was calculated. Its warp/weft ratio is $1: 1$, and its lay-up angle is $\pm 45^{\circ}$. The dimensions of the specimen are: $b=14.9 \mathrm{~mm}, h=1.6 \mathrm{~mm}, 2 a=70 \mathrm{~mm}$. The coordinates of the two strain gauges are: $x_{\mathrm{D}}=14.5 \mathrm{~mm}, x_{\mathrm{C}}=33 \mathrm{~mm}$. The creep load, $P$, is kept constant, i.e. $P=2 Q=78.48 \mathrm{~N}$.

The relaxation behaviour of specimen II- 6 was predicted. Its dimensions are: $b=15.2 \mathrm{~mm}, h=1.58 \mathrm{~mm}$ and $2 a=64.0 \mathrm{~mm}$. The deflexion of the central point of the beam was kept constant and $f_{\max }=1.363 \mathrm{~mm}$.

In the preliminary tests, the material initial elastic moduli were measured at $50^{\circ} \mathrm{C}$, with $E=8.34 \mathrm{GPa}$ and $G=0.18 \mathrm{GPa}$. The five constants in eqn (1) were determined through the procedure described above, as: $A=21.7926 \times 10^{-6}, \quad B=6.4796 \times 10^{-6}, \quad m=2.03558, \quad n=1.8061$, $q=0 \cdot 3446 / \mathrm{min}$.

Figure 8 shows the comparison between the experimental creep deflexion with that predicted by eqns (23) and (47). The predicted line of eqn (23) is much closer to the experimental one than that of eqn (47). 


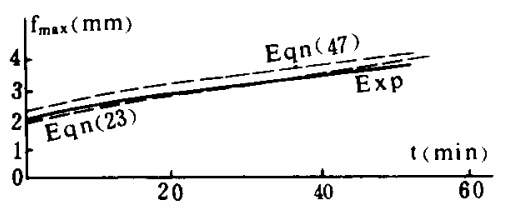

Fig. 8. Comparison of the predicted and measured creep strain curves. (-) Experimental; (-- ) predicted by eqn $(23) ;(-\cdot-\cdot)$ predicted by eqn (47).
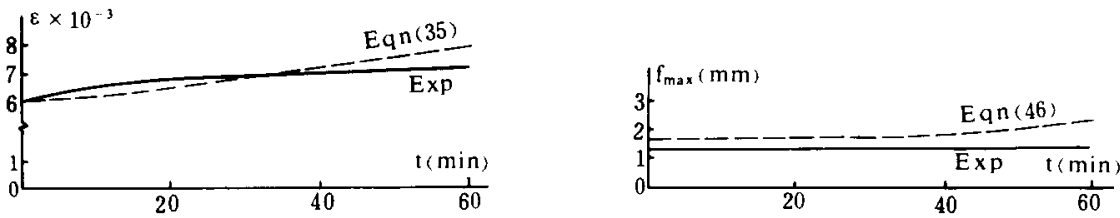

Fig. 9. Comparison between (---) predicted and $(\longrightarrow)$ measured relaxation

Fig. 10. Comparison between (---) predicted and (-) measured relaxation deflexion curves.

Inaccuracy comes from the fact that the nonlinearity of eqn (2) has been linearized in deriving eqn (47).

Figures 9 and 10 are curves of the relaxation strain and relaxation deflexion. The tendency of the increasing deviation of predicted curve from the experimental one is due to the nonlinear effect of the relaxation behaviour. For a long-term prediction, the linearization assumption is not feasible. Figure 10 also reveals the fact that the nonlinear effect becomes more important with time.

\section{CONCLUDING REMARKS}

The eight types of glass fibre fabric reinforced epoxy laminates were tested to examine the creep and relaxation behaviours. The creep deflexion and strains, and the relaxation load and strains were measured. It was found that the viscoelastic effect is significant and has to be taken into consideration in design of the structures made of these materials.

An attempt to predict the creep and relaxation behaviour was made. The predicted results were compared with the measured ones and found to be reasonably good, but the linearization assumption used in the analysis for relaxation deflexion has considerable limitations.

\section{ACKNOWLEDGEMENT}

This work was supported by The National Science Foundation and a special grant of the Chinese Academy of Sciences. 


\section{REFERENCES}

1. Zhang, S. Y. \& Kitching, R., Experimental relaxation behaviour of chopped strand mat glass fibre reinforced polyester beams in three-point bending. Composite Structures, 7 (1987) 59-76.

2. Zhao, Z., On the problem of nonlinear creeps. Acta Mechanica Sinica, 3 (1959) 325-34 (in Chinese).

3. Pao, Y. H. \& Marin, J., Deflection and stresses in beams subjected to bending and creep. J. Appl. Mech., Trans. ASME, 19 (1952) 478-84. 